\title{
Slow viruses and the immune system in the pathogenesis of local tissue damage in Sjögren's syndrome
}

\author{
George K Papadopoulos, Haralampos M Moutsopoulos
}

Primary Sjögren's syndrome is a systemic autoimmune disease characterised by intense focal lymphocytic infiltration of exocrine glands, accompanied by B lymphocytic hyperreactivity that can lead to B cell lymphomas. ${ }^{1}$ Hypotheses about the pathogenesis of the syndrome have changed with our increasing knowledge of basic biology and immunology. It has been suggested that hormonal, genetic, environmental, and stress factors set up several immune functional abnormalities, especially in the peripheral suppressor cell compartment of patients with primary Sjögren's syndrome. ${ }^{2} \mathrm{We}$ present here a number of new findings suggesting interplay between slow viruses in the epithelial cells of the exocrine glands and the immune system of patients with primary Sjögren's syndrome.

The immunological picture

Careful histopathological studies of exocrine glands of patients with primary Sjögren's syndrome show that infiltration of the glands is gradual and that there are few antigen presenting monocytes among the infiltrating cells. ${ }^{3-5}$ Most of the infiltrate comprises $\mathrm{T}$ cells, yet there is a distinct presence (about $20 \%$ of the total) of B cells $^{6}$ and few natural killer cells. Most of the T cells belong to the CD4+ helper-inducer category, while the remainder express the CD8 phenotype.$^{67}$ Most of these T cells are activated as they express DR antigens on their surface. These $T$ cells have been shown to secrete in vitro large quantities of interleukin $2^{8}$ and $\gamma$ interferon. ${ }^{7}$ The activated CD4+ $T$ cell population in the infiltrate consists of about two thirds memory cells (4B4+) and one third naive or suppressor-inducer $(2 \mathrm{H} 4+)$ cells. ${ }^{9}$ The former are thought of as the actual helperinducer cells for the growth and differentiation of B lymphocytes into antibody secreting cells. The $B$ lymphocytes in the exocrine gland infiltrates of patients with primary Sjögren's syndrome have been shown to secrete large amounts of immunoglobulin, rheumatoid factor, and various antibodies, such as anti-Ro and anti-La. ${ }^{10}$ These factors indicate an in situ immune response with a significant autoantigen specific component. The lack of monocytesthe classical antigen presenting cells-in the lesion makes this proposition problematical. On the other hand, the epithelial cells in the infiltrated exocrine glands show hyperexpression of HLA-A, B, and C antigens, as well as de novo inappropriate expression of HLA-DR antigens on their surface. Since $\gamma$ interferon has been shown to induce expression of both classes of histocompatibility antigens on the surface of epithelial and other cells one is faced here with the chicken and egg problem-that is, whether the DR expression and possible antigen presentation by epithelial cells predates or is a consequence of the lymphocytic infiltration. Furthermore, the infiltrating lymphocytes express the lymphocyte function associated antigen-1 (LFA-1) on their surface. ${ }^{9}$ This antigen, found on activated lymphocytes, binds to the intracellular adhesion molecule (ICAM-1) found on the surface of epithelial and endothelial cells. This adhesion molecule, also found on $T$ lymphocytes, and CD-2 are ligand for LFA-1 and LFA-3 respectively. The latter belongs to a group of proteins known as integrins with an important function in cell to cell contact. ${ }^{11} 12$ The presence of LFA-3 and ICAM-1 on the epithelial cells of the infiltrated exocrine glands in primary Sjögren's syndrome has yet to be established.

We need to determine, therefore, the causative agent for the de novo DR expression and the class I major histocompatibility complex (MHC) hyperexpression on the epithelial cells of the infiltrated exocrine glands of patients with primary Sjögren's syndrome. This was investigated indirectly by examining the conjunctival epithelial cells of patients with keratoconjunctivitis sicca and Sjögren's syndrome. Little lymphocytic infiltration of conjunctival epithelial cells was found in those conditions. There was, however, a substantially increased expression of MHC class I antigens and inappropriate expression of DR antigens on the conjunctival epithelial cells. ${ }^{13}$ Similar findings have been reported for viral and allergic conjunctivitis. ${ }^{13}$ As no $\gamma$ interferon was detected by the authors they concluded that this heightened expression of HLA-A, B, C and DR antigens was not due to lymphocytic infiltration and consequent $\gamma$ interferon secretion.

An additional and intriguing finding from the above set of patients with primary Sjögren's syndrome was that the 'nuclear' La autoantigen is found in the cytoplasm and on the membrane of the epithelial conjunctival cells, ${ }^{13}$ in contrast with such cells from normal controls, in whom the $\mathrm{La}$ antigen is present in minute amounts 
only in the nucleus. It is therefore possible to have antigen presentation of autoantigens, such as $\mathrm{La}$, through the de novo expressed DR antigens on epithelial cells. As these changes in behaviour of the epithelial cells occur without any lymphocytic infiltration we must assume that other changes-for example, hyperexpression of ICAM-1 and LFA-3 on proximal endothelial and epithelial cells-promote the infiltration of lymphocytes into the lesion. The stage is thus set for an in situ immune response, with epithelial cells of the exocrine glands presenting antigen to infiltrating $T$ cells, which in turn become activated and secrete various cytokines, thus activating B lymphocytes to secrete antibodies. These autoantibodies-for example, anti-La-may act directly in a stimulatory or cytotoxic fashion against the epithelial cells.

Are retroviruses responsible for the initiation of the observed tissue damage?

Having seen how the local autoimmune response might be initiated and perpetuated, we may wonder about the cause of the heightened HLA antigen expression which, by analogy with observations in conjunctival epithelial cells, must precede lymphocytic infiltration into the exocrine glands. A clue to its possible origin comes from several recent studies-one of animals and the others of humans. Transgenic mice bearing the tax gene from human $\mathrm{T}$ cell leukaemia/lymphoma virus type I (HTLV-I) produced a histopathological picture in the salivary and lachrymal glands resembling that of primary Sjögren's syndrome: initially, epithelial cells proliferate, followed by gradual infiltration of lymphocytes and plasma cells and, finally, gradual destruction of the acinus. ${ }^{14}$ The extent of histopathological change in various glands of these transgenic mice correlated directly with the concentration of the tax protein expressed in the nuclei of the epithelial cells. The tax protein is a trans-acting gene activator that in transfected $T$ lymphocytes increases the expression of the genes for interleukin 2, interleukin 2 receptor, interleukin 3, and granulocyte/monocyte colony stimulating factor. What is also obvious from this study is that the HTLV-I virus is tropic for the ductal epithelium of the salivary and lachrymal glands.

Now, a report from Japan has shown that Epstein-Barr virus and the adult $T$ cell leukaemia derived factor were present in the salivary glands of 10 out of 11 patients with Sjögren's syndrome. ${ }^{15}$ No HTLV-I was detected in these samples. Although the role of Epstein-Barr virus in activating $B$ lymphocytes is well known, one cannot at present postulate infection of the cells of the salivary glands by this virus.

In another study from the United Kingdom an endogenous retroviral sequence was detected in the salivary gland epithelium of $32 \%$ of patients with Sjögren's syndrome. ${ }^{16}$ The serological findings of the positive patients indicated the combined features of Sjögren's syndrome and rheumatoid arthritis. Although the original detection was performed with a monoclonal antibody to the HTLV-I p19 protein, sub- sequent work with synthetic peptides has shown that the reactive epitope has extensive homology with the endogenous retroviral agent HRES $1 / 1 .{ }^{17}$ Thus serum samples from patients with Sjögren's syndrome with high titres to HTLV-I peptides also show high titres against HRES 1/1 peptides. ${ }^{16}$

Perhaps significantly, in another study about $30 \%$ of patients with primary Sjögren's syndrome had serum antibodies that reacted with capsid antigen, p24/25, the major capsid protein of HIV, and a lower proportion of patients to the Gag protein of HIV. ${ }^{18}{ }^{19}$ This is in contrast with a $1-4 \%$ prevalence of such antibodies in the serum samples of healthy controls matched for age. This significant finding awaits confirmation from other laboratories.

Recently, a putative viral agent infecting the salivary glands of patients with primary Sjögren's syndrome was identified by culturing extracts from lip biopsy specimens of patients with RH9 lymphoblastoid cells. A type A retroviral particle was identified in two of six such patients studied. ${ }^{20}$ This particle is distinguishable from the HIV particle by several physicochemical and ultrastructural criteria. In line with the above is the finding that the oncogene c-myc is expressed in epithelial cells of infiltrated salivary glands of patients with primary Sjögren's syndrome. The activation of the oncogene may arise from any of several agents, possibly a retrovirus. ${ }^{21}$

These findings bristle with implications about the pathogenesis of primary Sjögren's syndrome. It seems that this type of retroviral infection could cause significant changes in the behaviour of the epithelial cells of exocrine glands, such as de novo expression of HLA-DR antigens, expression of $\mathrm{La}$ antigen on their surface, and heightened expression of lymphocytic adhesion molecules on the proximal endothelium. It may thus mean that retroviral infection of exocrine epithelial cells in subjects with the 'right' genetic and environmental substratum makes infiltration of immunocytes and development of a localised autoimmune response inevitable, with all the pathological consequences that ensue.

As work continues other retroviral agents may also be reported. It is necessary to know which, if any, of the retroviruses detected cause disease and which are merely an epiphenomenon. This might be tested by a prospective time course study, recording the precise sequence of events and assessing the importance of each component in the pathogenesis of the disease.

It may also be that the putative retroviral agent(s) possesses epitopes that are similar to epitopes of self antigens, thus converting an immune defence process into an autoimmune response. There are several cases where such a relation has been postulated as, for example, in the case of La autoantigen (amino acids 88-101) and the gag protein of feline sarcoma virus. ${ }^{22}$ The whole subject of cross reactivity between viral antigens and self antigens and its relation to autoimmunity was examined in a recent publication, ${ }^{23}$ but before any further hypotheses are suggested the precise identity of the retroviral entity must be established. 
We thank Mr G E Papanikolaou for excellent secretarial assistance.

1 Moutsopoulos H M, Talal N. New developments in Sjögren's syndrome. Curr Opin Rheumatol 1989; 1: 332-8.

2 Tzioufas A G, Moutsopoulos H M. Siögren's syndrome. In: Khamashta M A, Font J, Hughes G R V, eds. Connective tissue disease: selected topics. In press.

3 Daniels T E. Labial salivary gland biopsy in Sjögren's syndrome: assessment as a diagnostic criterion in 362 suspected cases. Arthritis Rheum 1984; 27: 147-56.

4 Lindahl G, Hedfors E, Klareskog L, Forsum U. Epithelial HLA-DR expression and T-lymphocyte subsets in salivary glands in Sjögren's syndrome. Clin Exp Immunol 1985; 61: 475-82.

5 Moutsopoulos H M, Hooks J J, Chan C C, Dalavanga Y A, Skopouli F N, Detrick B. HLA-DR expression in labial salivary gland tissues in Sjögren's syndrome. Ann Rheum Dis 1986; 45: 677-83.

6 Fox R, Carstens S, Fong S, Robinson C A, Howell P, Vaughan J H. Use of monoclonal antibodies to analyze peripheral blood and salivary gland lymphocyte subsets in Siögren's syndrome. Arthritis Rheum 1982; 25: 419-22.

7 Dalavanga Y A, Drosos A A, Moutsopoulos H M. Labial salivary gland immunopathology in Sjögren's syndrome. Scand 7 Rheumatol 1986; suppl 61: 67-70.

8 Fox R I, Theophilopoulos A N, Altman A A. Production of interleukin-2 (IL-2) by salivary gland lymphocytes in Sjögren's syndrome. Detection of reactive cells by using Sjogren's syndrome. Detection of reactive cells by using antibodies directed to synthe

9 Skopouli F N, Fox P C, Galanopoulou V, Atkinson J C, Jaffe B C, Moutsopoulos H M. T cell subpopulations in the labial minor salivary gland histopathologic lesion of Sjögren's syndrome. F Rheumatol 1991; 18: 210-4.

10 Talal N, Asofsky R, Lightbody P. Immunoglobulin synthesis by salivary gland lymphoid cells in Siögren's syndrome. F Clin Invest 1970; 49: 49-54.

11 Albelda S M, Buck C A. Integrins and other adhesion molecules. FASEB f 1990; 4: 2868-80.
12 Springer T A. Adhesion receptors of the immune system. Nature 1990; 346: 425-34.

13 Giannopoulos D, Roncin S, Pennec Y L, et al. Conjunctival epithelial cells of patients with primary Sjögren's syndrome express DR and La antigens (abstract). Clin Rheumatol 1990; 9: 556

14 Green J B, Hinricks S H, Vogel J, Jay G. Exocrinopathy resembling Siögren's syndrome in HTLV-1 tax transgenic mice. Nature 1989; 341: 72-4.

15 Saitp I, Nishimura S, Kudo, et al. Adult T-cell leukemiaderived factor (ADF) in Sjögren's syndrome (abstract). Clin Exp Rheumatol 1991; 9: 336.

16 Shattles W R, Venables P J W, Brookes S M, Maini R N Detection of endogenous retroviral sequences in salivary gland epithelium in patients with Siögren's syndrome (abstract). Clin Exp Rheumatol 1991; 9: 337.

17 Perl A, Rosenblatt J D, Chen I S Y, et al. Detection and cloning of new HTLV-related endogenous sequences in man. Nucl Acids Res 1989; 17: 6841-54.

18 Talal N, Dauphinee M J, Dang H, Alexander S S, Hart D J Garry R F. Detection of serum antibodies to retroviral proteins in patients with primary Sjögren's syndrome (autoimmune exocrinopathy). Arthritis Rheum 1990; 33: 774-81.

19 Talal N, Garry R F, Schure P H, et al. A conserved idiotype and antibodies to retroviral proteins in systemic lupus erythematosus. $\mathcal{F}$ Clin Invest 1990; 85: 1866-71.

20 Garry R F, Fermin C D, Hart D J, Alexander S S, Donehower $L A$, Luo-Zhang $H$. Detection of a human intracisternal A-type retroviral particle antigenically related to HIV. Science 1990; 250: 1127-9.

21 Skopouli F N, Galanopoulou V, Siouna-Fatourou H, et al. c-myc mRNA in labial salivary glands of patients with Siögren's syndrome (abstract). Clin Rheumatol 1990; 9: 558 .

22 Kohsaka H, Yamamoto K, Fujii H, et al. Fine epitope mapping of the human SSB/La protein. Identification of a distinct autoepitope homologous to a viral gag polyprotein. 7 Clin Invest 1990; 85: 1566-74.

23 Oldstone M B A, ed. Molecular mimicry: cross-reactivity between microbe and host proteins as a cause of autoimmunity. Curr Top Microbiol Immunol 1989; 145: 1-135. 